\title{
Scalar Conservation Laws with Discontinuous Flux Function: I. The Viscous Profile Condition
}

\section{S. Diehl}

Department of Mathematics, Lund Institute of Technology, P.O. Box 118, S-221 00 Lund, Sweden, E-mail: diehl@maths.lth.se

Received: 22 November 1994

\begin{abstract}
The equation $\frac{\partial u}{\partial t}+\frac{\partial}{\partial x}(H(x) f(u)+(1-H(x)) g(u))=0$, where $H$ is Heaviside's step function, appears for example in continuous sedimentation of solid particles in a liquid, in two-phase flow, in traffic-flow analysis and in ion etching. The discontinuity of the flux function at $x=0$ causes a discontinuity of a solution, which is not uniquely determined by the initial data. The equation can be written as a triangular $2 \times 2$ non-strictly hyperbolic system. This augmentation is non-unique and a natural definition is given by means of viscous profiles. By a viscous profile we mean a stationary solution of $u_{t}+\left(F^{\delta}\right)_{x}=\varepsilon u_{x x}$, where $F^{\delta}$ is a smooth approximation of the discontinuous flux, i.e., $H$ is smoothed. In terms of the $2 \times 2$ system, the discontinuity at $x=0$ is either a regular Lax, an under- or overcompressive, a marginal under- or overcompressive or a degenerate shock wave. In some cases, depending on $f$ and $g$, there is a unique viscous profile (e.g. undercompressive and regular Lax waves) and in some cases there are infinitely many (e.g. overcompressive waves). The main purpose of the paper is to show the equivalence between a previously introduced uniqueness condition for the discontinuity of the solution at $x=0$ and the viscous profile condition.
\end{abstract}

\section{Introduction}

The scalar conservation law with discontinuous flux function

$$
\frac{\partial u(x, t)}{\partial t}+\frac{\partial}{\partial x}\left(F^{0}(u(x, t), x)\right)=0, \text { where } F^{0}(u, x)= \begin{cases}f(u), & x>0 \\ g(u), & x<0\end{cases}
$$

arises in several applications, for example in continuous sedimentation of solid particles in a liquid, see Diehl [3] and Chancelier et al. [1], in two-phase flow in porous media, see Gimse and Risebro [6], in traffic-flow analysis, see Mochon [11], and in ion etching in the fabrication of semiconductor devices, see Ross [13]. In these applications (except perhaps in traffic flow) the flux functions $f$ and $g$ are non-convex. The Cauchy problem for a more general equation than (1.1), including a point source $s(t)$ at $x=0$, has been analysed by the author in [2]. Generally, a solution of (1.1) 
contains a discontinuity along the $t$-axis and curves of discontinuity that go into and emanate from it. For given initial data $u(x, 0)$ there is usually a whole set of pairs of boundary values along $x=0$ that can appear in functions $u(x, t)$ satisfying (1.1). In [2] Condition $\Gamma$ was introduced, which implies that only one pair of this set is used. Under some regularity assumptions, existence and uniqueness results are obtained in [2]. Condition $\Gamma$ is a generalization of the classical entropy condition by Oleinik [12] and it is, in [2], justified by studying an extension of Godunov's [7] numerical method. In Sect. 2 we review some notation and results from [2].

In Subsect. 3.1 we define a viscous profile (corresponding to the discontinuity at $x=0$ of (1.1)) as a stationary solution of $u_{t}+\left(F^{\delta}\right)_{x}=\varepsilon u_{x x}$, where $F^{\delta}(u, \cdot)$ (with $\delta>0)$ is a smooth approximation of $F^{0}(u, \cdot)$.

Equation (1.1) can be augmented to a $2 \times 2$ triangular non-strictly hyperbolic system, with flux function independent of $x$, by adding a scalar conservation law taking care of the discontinuity of $F^{0}(u, \cdot)$, cf. Gimse and Risebro [5]. The flux function of this added scalar equation is not uniquely determined. Requiring that the viscous profile of the $2 \times 2$ system should be the same as the one described in the preceding paragraph, an explicit formula for the added flux function in terms of the smoothing of $F^{0}(u, \cdot)$ is given in Subsect. 3.2. In this context, the Riemann problem is also treated by Gimse and Risebro [5] and, for $n \times n$ triangular systems, by Holden and Høegh-Krohn [8].

Depending on $f$ and $g$, there are three qualitatively different cases of shock wave and viscous wave. In Subsect. 3.3 we relate this natural division to the over-, undercompressive waves, etc. of the $2 \times 2$ system described above.

Sect. 4 is devoted to showing that Condition $\Gamma$ is equivalent to the viscous profile condition in the sense that the concentration to the left $\left(u^{-}\right)$and to the right $\left(u^{+}\right)$of the $t$-axis obtained by Condition $\Gamma$ are the only ones such that there exists a viscous profile between $u^{-}$and $u^{+}$. This "equivalence" can be seen as a generalization of the "equivalence" between Oleinik's entropy condition and the viscous profile condition for the scalar equation $u_{t}+f(u)_{x}=0$. As is well known, there exist shock waves, satisfying Oleinik's entropy condition, which are unstable when including diffusion, see Example 4.1. In the same way, there exist shocks along $x=0$ in the case $f \neq g$, satisfying Condition $\Gamma$, which are unstable when including diffusion, see Examples 4.2 and 4.3 .

We could have let $\delta=0$ (and $\varepsilon>0$ ) when examining the uniqueness conditions, see Subsect. 4.4. However, in the applications referred to above $\delta>0$ may provide better models. Compare with the fact that $\varepsilon>0$ usually provides better models than $\varepsilon=0$, that is, there is usually some viscosity or diffusion. For example, in continuous sedimentation the discontinuous flux function appears due to a feed inlet to a sedimentation tank. Taking $\delta=0$ corresponds to a point source and this is clearly not the case in reality, where the inlet on one hand has a positive cross-sectional area, on the other causes mixing near $x=0$. Furthermore, we want to present the qualitatively different viscous profiles that appear when $\delta>0$, since some stability results of these are presented in the subsequent paper [4]. It is interesting to note that some viscous profiles are non-monotone. This occurs mainly for overcompressive shocks, but may occur even for a regular Lax shock wave, see Fig. 12.

\section{Scalar Conservation Laws with Discontinuous Flux Function}

For solutions of the Cauchy problem, with $h \in C^{2}$, 


$$
\begin{array}{ll}
u_{t}+h(u)_{x}=0, & x \in \mathbb{R}, t>0, \\
u(x, 0)=u_{0}(x), & x \in \mathbb{R},
\end{array}
$$

the jump condition

$$
x^{\prime}(t)=S\left(u^{x+}, u^{x-}\right)
$$

and the entropy condition

$$
S\left(v, u^{x-}\right) \geq S\left(u^{x+}, u^{x-}\right), \quad \forall v \text { between } u^{x-} \text { and } u^{x+}
$$

by Oleinik [12] are assumed to hold, where $x=x(t)$ is a curve of discontinuity, $S(\alpha, \beta)=\frac{h(\alpha)-h(\beta)}{\alpha-\beta}$ for $\alpha \neq \beta$ and $u^{x \pm}=u(x(t) \pm 0, t)$.

When $f \neq g$ in (1.1) the new feature is the discontinuity that appears at $x=0$. We assume that $f, g \in C^{2}$ have at most a finite number of stationary points. For $u$ piecewise smooth we introduce

$$
\begin{aligned}
& u_{ \pm}(t)=\lim _{\delta \searrow 0} u( \pm \delta, t), \\
& u^{ \pm}(t)=\lim _{\varepsilon \searrow 0} u_{ \pm}(t+\varepsilon) .
\end{aligned}
$$

The order of the limit processes is significant for example when a discontinuity emerges from the $t$-axis. The Cauchy problem of (1.1) can be written

$$
\begin{array}{ll}
u_{t}+f(u)_{x}=0, & x>0, t>0, \\
u_{t}+g(u)_{x}=0, & x<0, t>0, \\
f\left(u^{+}(t)\right)=g\left(u^{-}(t)\right), & t>0, \\
u(x, 0)=u_{0}(x), & x \in \mathbb{R} .
\end{array}
$$

This problem has been analysed in [2] and we refer to that paper for a full description including proofs and examples. To be able to construct a solution by the method of characteristics, one needs to find boundary functions on either side of the $t$-axis. These functions should satisfy the third equation of (2.3), i.e., the conservation law over the $t$-axis. It turns out that these boundary functions are not only difficult to obtain but they are also non-unique. Even if $f$ and $g$ are linear functions, the boundary functions are not uniquely determined by the third equation of (2.3) as the following example shows.

Example 2.1. Let $f(u)=u, g(u)=-u$ and $u(x, 0)=\left\{\begin{array}{ll}u_{l}, & x<0 \\ u_{r}, & x>0\end{array}\right.$, see Fig. 1. Independently of the values $u^{ \pm}$, with $f\left(u^{+}\right)=g\left(u^{-}\right)$, the characteristics always emanate from the $t$-axis. Hence there exist infinitely many possibilities to construct a solution. Two possible choices of $u^{+}$and $u^{-}$are shown in Fig. 1, where the jump from $u^{-}$ to $u^{+}$corresponds to a horizontal dotted line from the graph of $g$ to the graph of $f$. As is motivated in [2], of these solutions the one with $u^{-}=u^{+}=\bar{u}$ turns out to be the physically correct one. Another motivation will be given by the viscous profile analysis in Sect. 4.

In the region $x>0, t>0$, the concentration is determined by the equation $u_{t}+f(u)_{x}=0$. Consider the constant initial datum $u(x, 0)=u_{+}, x>0$. For what boundary values $\alpha$ is it possible to construct a solution satisfying $u^{+}(t) \equiv \alpha$ ? Any 

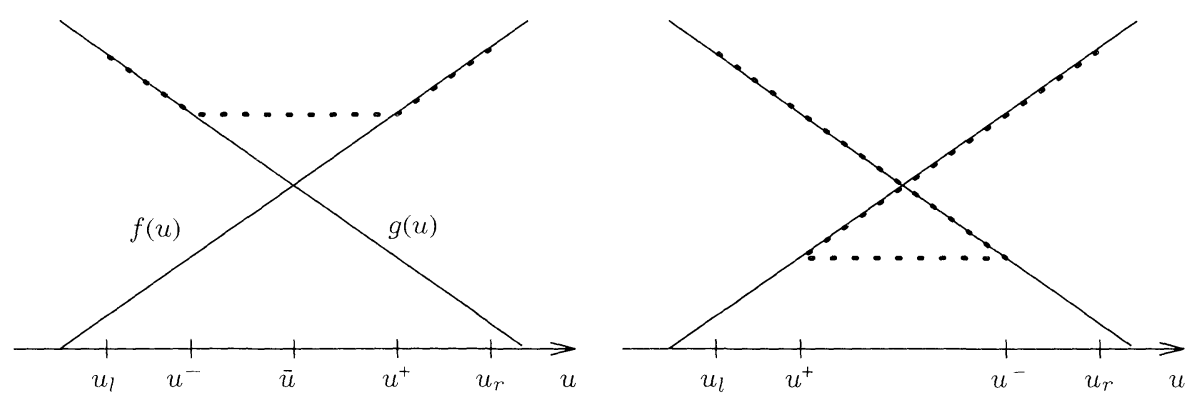

Fig. 1. (Example 2.1) There are infinitely many choices of $u^{--}$and $u^{+}$.

such solution is obtained by taking the restriction to the region $x>0, t>0$, of the solution of the Riemann problem:

$$
\begin{aligned}
& u_{t}+f(u)_{x}=0, \quad x \in \mathbb{R}, t>0, \\
& u(x, 0)=\left\{\begin{array}{ll}
\alpha, & x<0 \\
u_{+}, & x>0
\end{array} .\right.
\end{aligned}
$$

The general solution of (2.4) (with the entropy condition (2.2)) is of the form $u(x / t)$ and can be described by a cone in the $x$-t-plane, to the left and right of which the solution is identically equal to $\alpha$ and $u_{+}$, respectively. Inside the cone the solution is a monotone function of $x / t$, built up of rarefaction waves separated by discontinuities. If the cone lies to the right of the $t$-axis, we clearly obtain $u^{+}(t) \equiv \alpha$. Generally, we define the set of possible $\alpha$ as

$$
P\left(f ; u_{+}\right)=\left\{\alpha: \text { the solution of }(2.4) \text { satisfies } u^{+}(0)=\alpha\right\} .
$$

This set is closely related to the function

$$
\hat{f}\left(u ; u_{+}\right)= \begin{cases}\min _{v \in\left[u, u_{+}\right]} f(v), & u \leq u_{+} \\ \max _{v \in\left[u_{+}, u\right]} f(v), & u>u_{+}\end{cases}
$$

see Fig. 2. The function $\hat{f}\left(\cdot ; u_{+}\right)$is non-decreasing and its graph consists of increasing parts separated by plateaus, where the function is constant. At the increasing parts, i.e. when $u \in P, \hat{f}\left(u ; u_{+}\right)=f(u)$ holds. Analogously we introduce the set $N\left(g ; u_{-}\right)$ of possible boundary values on the left side of the $t$-axis and the corresponding nonincreasing function $\check{g}\left(\cdot ; u_{-}\right)$. In Example 2.1 above $\hat{f}=f, \check{g}=g$ and $P=N=\mathbb{R}$ hold, hence all values are possible for $u^{+}$and $u^{-}$. As was mentioned above, the choice $u^{+}=u^{-}=\bar{u}$, which is the intersection of the graphs of $\hat{f}$ and $\check{g}$, is physically significant.

We shall now try to motivate a uniqueness condition for (2.3) by generalizing the entropy condition (2.2) for problem (2.1), which we can consider as a special case of (2.3) with $f=g=h$. Consider initial data of (2.1) which produce a discontinuity with zero speed between $u^{-}$and $u^{+}$. According to the jump condition and the entropy condition (2.2) the graph of $h$ lies above the horizontal line (the shock speed is zero) through the points $\left(u^{-}, h\left(u^{-}\right)\right)$and $\left(u^{+}, h\left(u^{+}\right)\right)$, see Fig. 3 (left). This is equivalent 

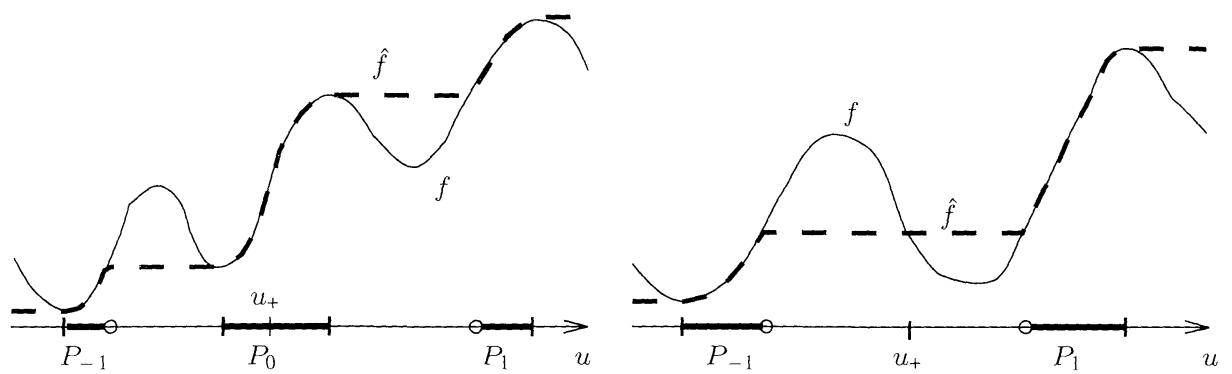

Fig. 2. The graphs of $f$ (solid) and the two main possibilities for $\hat{f}\left(\cdot ; u_{+}\right)$(dashed). The set $P=\bigcup_{\imath} P_{\imath}$, where $P_{0}=\left\{u_{+}\right\}$in the right-hand plot. Note that some intervals are half-open by the definition of $P$.
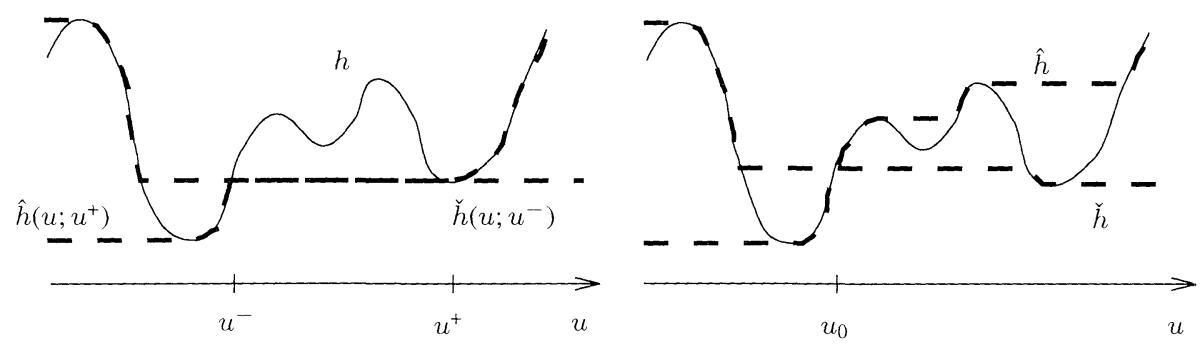

Fig. 3. The entropy condition (2.2) can be described by the intersection of $\hat{h}$ and $\check{h}$.

to

$$
h\left(u^{+}\right)=h\left(u^{-}\right)=\min _{u^{-} \leq v \leq u^{+}} h(v) .
$$

By definition $\hat{h}\left(u^{-} ; u^{+}\right)=\min _{u^{-} \leq v \leq u^{+}} h(v)=\breve{h}\left(u^{+} ; u^{-}\right) \equiv \gamma$, which is the flux level of the intersection of $\hat{h}$ and $\breve{h}$. The entropy condition (2.2) can thus be written (for zero-speed shocks)

$$
h\left(u^{+}\right)=h\left(u^{-}\right)=\gamma .
$$

Now suppose the initial datum of (2.1) is constant $u_{0}$. Then the unique entropy solution is this constant $u_{0}=u^{+}=u^{-}$. Again, $h\left(u_{0}\right)$ is the flux value of the intersection of $\hat{h}$ and $\breve{h}$, see Fig. 3 (right).

With the above discussion in mind we shall need the following notation in order to formulate Condition $\Gamma$, which involves the intersection of $\hat{f}$ and $\breve{g}$ and which can handle (2.3) as well. Let $u_{+}, u_{-} \in \mathbb{R}$ be given. The projection on the $u$-axis of the intersection of the graphs of $\hat{f}$ and $\breve{g}$ and its flux value, are denoted by

$$
\begin{aligned}
& \bar{U}=\bar{U}\left(u_{+}, u_{-}\right)=\left\{u \in \mathbb{R}: \hat{f}\left(u ; u_{+}\right)=\check{g}\left(u ; u_{-}\right)\right\}, \\
& \gamma=\hat{f}\left(\bar{U} ; u_{+}\right),
\end{aligned}
$$

see Fig. 4. Since $\hat{f}$ is non-decreasing and $\check{g}$ is non-increasing, $\bar{U}$ is an interval. When the set $\bar{U}$ only consists of one point, this point is denoted by $\bar{u}$. Further introduce the set of pairs 


$$
\Gamma\left(u_{+}, u_{-}\right)=\left\{(\alpha, \beta) \in \mathbb{R}^{2}: f(\alpha)=g(\beta)=\gamma\right\}
$$

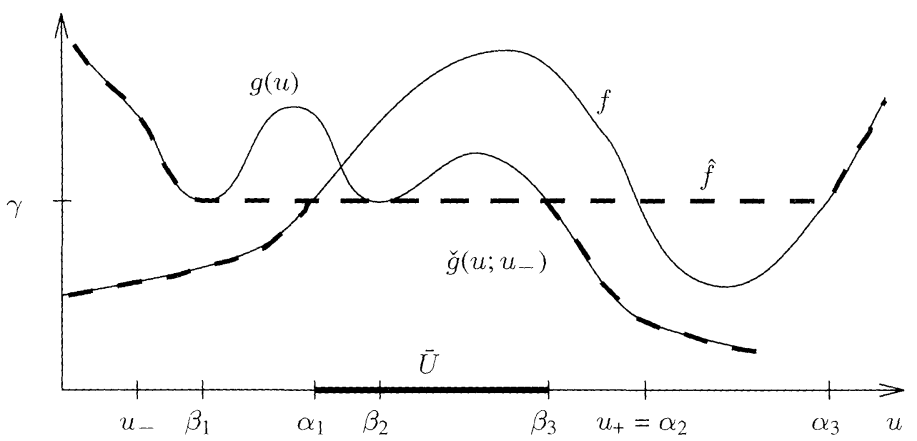

Fig. 4. An example of the set $\bar{U}$. The dashed line from $\beta_{1}$ to $\beta_{3}$ is a plateau of $\check{g}\left(\cdot ; u_{-}\right)$, and from $\alpha_{1}$ to $\alpha_{3}$ a plateau of $\hat{f}\left(\cdot ; u_{+}\right)$. Note that $\Gamma=\left\{\left(\alpha_{\imath}, \beta_{\jmath}\right): i, j=1,2,3\right\}$.

Condition $\Gamma$. For given $u_{+}, u_{-} \in \mathbb{R},\left(u^{+}, u^{-}\right) \in \Gamma\left(u_{+}, u_{-}\right)$holds.

Note that Condition $\Gamma$ reduces to (2.5) when $f=g=h, u^{+}=u_{+}$and $u^{-}=u_{\ldots}$. As can be seen from the example in Fig. 4 the set $\Gamma$ may consist of more than one pair. It can be shown, see [2], that for given initial data only one pair in $\Gamma$ can be used to start the construction of a solution. The correct choice depends on the behaviour of the solution in a neighbourhood of the $t$-axis. This also implies that, generally, boundary functions on either side of the $t$-axis can not be given beforehand globally in $t$. However, with the Riemann initial data $u_{0}(x)=\left\{\begin{array}{ll}u_{-}, & x<0 \\ u_{+}, & x>0\end{array}\right.$ in (2.3), with $u_{-}$ and $u_{+}$constant, boundary functions $\alpha$ and $\beta$ can be given uniquely beforehand as a function of $u_{-}$and $u_{+}$, see [2]. In fact, with the Riemann initial data, Condition $\Gamma$ reduces to the explicit form $(\alpha, \beta)=c\left(u_{+}, u_{-}\right)$, see Theorem 2.1 below, where the function $c$ is defined in the following proposition. Denote the set of intersecting ranges by

$$
I\left(u_{+}, u_{-}\right)=\hat{f}\left(\mathbb{R} ; u_{+}\right) \cap \check{g}\left(\mathbb{R} ; u_{-}\right) .
$$

Proposition 2.1. Let $u_{+}, u_{-} \in \mathbb{R}$ be given. If $I\left(u_{+}, u_{-}\right) \neq \emptyset$, then the set $\left(P\left(f ; u_{+}\right) \times\right.$ $\left.N\left(g ; u_{-}\right)\right) \cap \Gamma\left(u_{+}, u_{-}\right)$consists of exactly one pair and hence a function $c$ is well defined by

$$
c\left(u_{+}, u_{-}\right)=\left(u^{+}, u^{-}\right) \in(P \times N) \cap \Gamma .
$$

Proof. $I \neq \emptyset$ implies $\bar{U} \neq \emptyset$. Since the restrictions $\left.f\right|_{P}$ and $\left.g\right|_{N}$ are injective,

$$
\left.\left(u^{+}, u^{-}\right) \in(P \times N) \cap \Gamma \quad \Longleftrightarrow f\right|_{P}\left(u^{+}\right)=\left.g\right|_{N}\left(u^{-}\right)=\gamma
$$

uniquely determines $u^{+}$and $u^{-}$. 

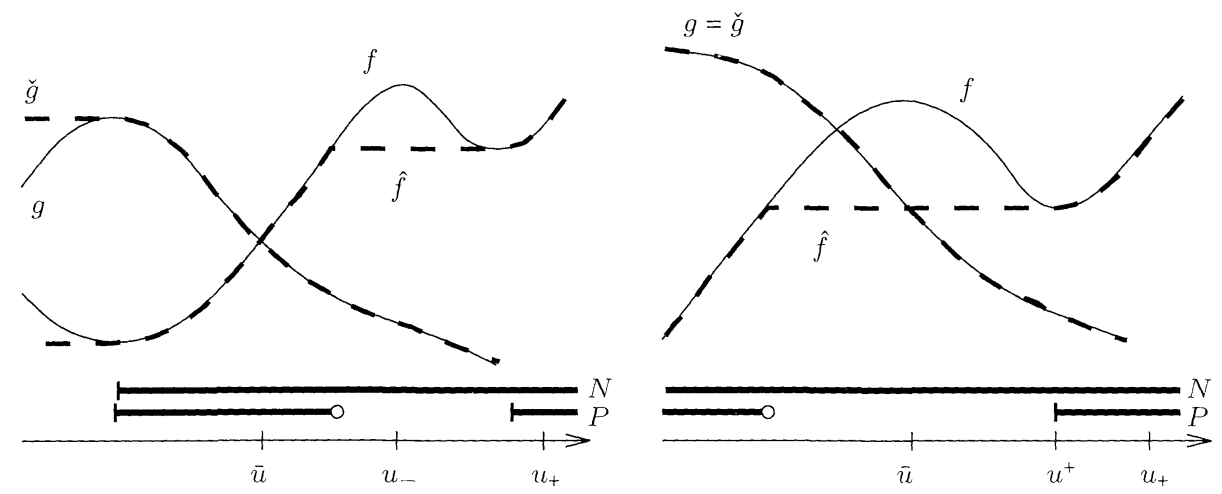

Fig. 5. Examples of Case 1 (left) and Case 2 a (right). The sets $N$ and $P$ are indicated above the $u$-axis.

Depending on the set $\bar{U}\left(u_{+}, u_{-}\right)$, the function $c$ yields that there are three qualitatively different types of discontinuity. This is reflected in the three different types of intersection of the graphs of $\hat{f}$ and $\breve{g}$, depending on whether none, one or two plateaus are involved.

Case 1. $\bar{u} \in N \cap P$, see Fig. 5 and Example 2.1. The function $c$ yields $u^{+}=u^{-}=\bar{u}$. Case $2 a . \bar{u} \in N \backslash P$, see Fig. 5.

Case $2 b . \bar{u} \in P \backslash N$ (symmetric to Case $2 \mathrm{a}$ ).

Case 3. $\bar{U}$ is infinite or $\bar{u} \in \complement(P \cup N)$, see Fig. 6 .
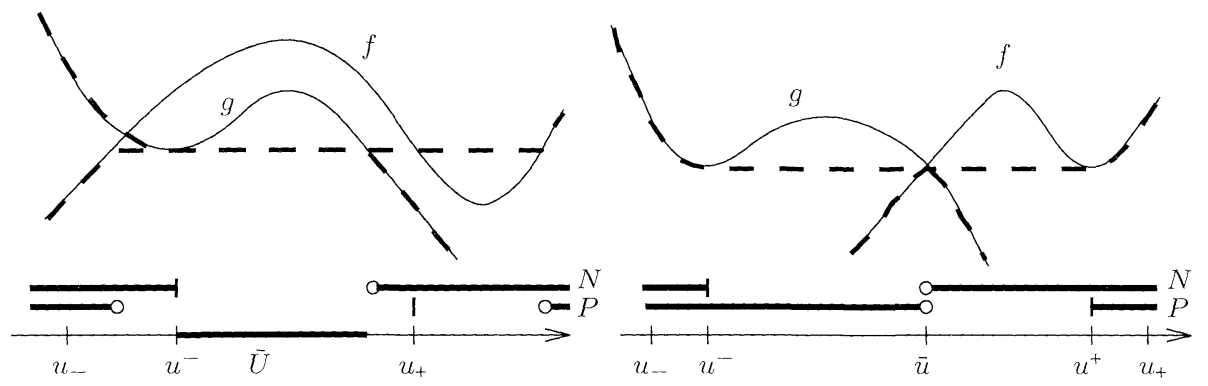

Fig. 6. Examples of Case 3: The set $\bar{U}$ is infinite (left) and $\bar{u} \in \mathbb{C}(P \cup N)$ (right). In the left figure $u^{+}=u_{+}$ holds.

Theorem 2.1. Given $u_{l}$ and $u_{r}$ with $I\left(u_{r}, u_{l}\right) \neq \emptyset$. Then the Riemann problem with discontinuous flux function

$$
\begin{aligned}
& u_{t}+f(u)_{x}=0, \quad x>0, t>0, \\
& u_{t}+g(u)_{x}=0, \quad x<0, t>0 \text {, } \\
& f\left(u^{+}(t)\right)=g\left(u^{-}(t)\right), \quad t>0, \\
& u(x, 0)= \begin{cases}u_{l}, & x<0 \\
u_{r}, & x>0\end{cases}
\end{aligned}
$$


has a unique solution which satisfies Condition $\Gamma$ for $t \geq 0$, is piecewise smooth and for which $u^{ \pm}(t)$ are piecewise monotone. The solution is of the form $u(x / t)$ and the constant boundary values are given by $\left(u^{+}, u^{-}\right)=c\left(u_{r}, u_{l}\right)$.

The proof can be found in [2]. Writing (2.6) as a $2 \times 2$ system, see Subsect. 3.2, the result on triangular $n \times n$ systems by Holden and Høegh-Krohn [8] yields that there exists a unique solution of (2.6) of the form $u(x / t)$, which satisfies the viscous profile condition. However, there are situations when Condition $\Gamma$ yields a (unique) solution, whereas no viscous profile exists between any of the possible states connecting a discontinuity, see Examples 4.2 and 4.3 below. The situation is analogous to the one involving Oleinik's entropy condition for the scalar equation $u_{t}+f(u)_{x}=0$, see Example 4.1 below.

\section{The Viscous Profiles}

\subsection{Definition.}

We shall now examine stationary wave solutions of (1.1) by smoothing the discontinuity in the flux function and by adding a viscosity term. Let $H$ denote Heaviside's step function and let $h$ be a scalar smooth function of one variable with $h^{\prime}(x) \equiv 0$ for $|x| \geq 1$ and $h(x)$ strictly increasing from 0 to 1 in $|x|<1$. Then $h(x / \delta) \rightarrow H(x)$ as $\delta \searrow 0$ in distribution sense and

$$
F^{\delta}(u, x) \equiv h(x / \delta) f(u)+(1-h(x / \delta)) g(u) \rightarrow F^{0}(u, x), \quad \delta \searrow 0
$$

We consider solutions of (1.1) as limit solutions of the parabolic equation

$$
u_{t}+\left(F^{\delta}(u, x)\right)_{x}=\varepsilon u_{x x}
$$

as $\delta$ and $\varepsilon \searrow 0$ with the same speed. Let $\delta$ and $\varepsilon$ be fixed and set $\xi=x / \varepsilon$. Assume that there is a smooth stationary solution $u(x, t)=v(\xi)$ of $(3.1)$, i.e., $\left(F^{\delta}(v, \varepsilon \xi)\right)_{\xi}=v^{\prime \prime}$. Of course $v$ also depends on $\delta$ but we do not write this out explicitly. We get

$$
v^{\prime}(\xi)=F^{\delta}(v(\xi), \varepsilon \xi)+C
$$

Assuming $v(\xi) \rightarrow u^{ \pm}$as $\xi \rightarrow \pm \infty$ and using the third equation of (2.3) we can determine the constant

$$
C=-f\left(u^{+}\right)=-g\left(u^{-}\right) .
$$

The dynamic equation becomes

$$
v^{\prime}(\xi)=h(\varepsilon \xi / \delta) f(v(\xi))+(1-h(\varepsilon \xi / \delta)) g(v(\xi))-g\left(u^{-}\right) \text {. }
$$

We call a solution $v$ of (3.2) a viscous profile for a discontinuity between $u^{-}$and $u^{+}$ of a solution of (1.1) if $v(\xi) \rightarrow u^{ \pm}$as $\xi \rightarrow \pm \infty$. 


\subsection{The Associated Non-Strictly Hyperbolic System.}

Equation (1.1) can be written as a $2 \times 2$ triangular system by adding a scalar equation having $H(x)$ as stationary solution, cf. Gimse and Risebro [5, 6]. Let $k(a)$ be a scalar function with $k(0)=k(1)=0, k(a) \geq 0$ for $a \in[0,1]$ and smooth in $(0,1)$. Then the Riemann problem

$$
\begin{aligned}
& a_{t}+k(a)_{x}=0, \quad x \in \mathbb{R}, t>0, \\
& a(x, 0)=H(x)
\end{aligned}
$$

has the stationary solution $a(x, t) \equiv H(x)$. Equation (1.1) may then be written as a system

$$
\left(\begin{array}{l}
u \\
a
\end{array}\right)_{t}+\left(\begin{array}{c}
a f(u)+(1-a) g(u) \\
k(a)
\end{array}\right)_{x}=\left(\begin{array}{l}
0 \\
0
\end{array}\right), \quad x \in \mathbb{R}, t>0 .
$$

The eigenvalues of the Jacobian of the flux matrix are

$$
\begin{aligned}
& \lambda_{1}(u, a)=a f^{\prime}(u)+(1-a) g^{\prime}(u), \\
& \lambda_{2}(u, a)=k^{\prime}(a),
\end{aligned}
$$

so generally (3.3) is a non-strictly hyperbolic system. The sense in which (1.1) and (3.3) are equivalent depends on the function $k$. Below we shall give a natural definition of $k$ by claiming that the viscous profile of (3.2) should be the same as the corresponding viscous profile of (3.3). The latter is usually defined by adding a small viscosity matrix times $\partial_{x x}$ of the state vector on the right-hand side of (3.3). It is common to let the viscosity matrix be diagonal and in our case it would be unnatural to assume any coupling of viscosity effects between $u$ and $a$ with the following motivation. The second equation of (3.3) describes a given physical configuration, which does not depend on $u$ at all. Thus we add only the term $\delta a_{x x}$ to the second equation. The solution $a$ (with or without the term $\delta a_{x x}$ ) should affect $u$ in the first equation only via the flux function, by the original formulation of the problem. Thus we add only the term $\varepsilon u_{x x}$ to the first equation, leading to the parabolic system

$$
\left(\begin{array}{l}
u \\
a
\end{array}\right)_{t}+\left(\begin{array}{c}
a f(u)+(1-a) g(u) \\
k(a)
\end{array}\right)_{x}=\left(\begin{array}{l}
\varepsilon u \\
\delta a
\end{array}\right)_{x x}, \quad x \in \mathbb{R}, t>0 .
$$

Assume that this system admits stationary solutions $u(x, t)=u(\xi)=u(x / \varepsilon)$ and $a(x, t)=a(\rho)=a(x / \delta)$, with $u(\xi) \rightarrow u^{ \pm}$as $\xi \rightarrow \pm \infty$ and $a(\rho) \rightarrow\left\{\begin{array}{l}1 \\ 0\end{array}\right.$ as $\rho \rightarrow \pm \infty$. Then we obtain the dynamic system, with $\tilde{a}(\xi)=a(\varepsilon \xi / \delta)$,

$$
\left\{\begin{array}{l}
\dot{u}=\tilde{a} f(u)+(1-\tilde{a}) g(u)-g\left(u^{-}\right) \\
\dot{\tilde{a}}=k(\tilde{a})
\end{array}, \quad \text { where } \cdot \equiv \frac{d}{d \xi} .\right.
$$

Note that $\left(u^{-}, 0\right)$ and $\left(u^{+}, 1\right)$ are fixed points of $(3.5)$.

Requiring that (3.2) and (3.5) should describe the same viscous profile yields

$$
\tilde{a}(\xi)=h(\varepsilon \xi / \delta) \in[0,1], \quad \xi \in \mathbb{R} .
$$


It is natural to use the same $\varepsilon$ and $\delta$ of (3.2) and (3.5), so that $a \equiv h$. For $|x|<1$, $h(x)$ is strictly increasing. Hence, for $|\xi|<\delta / \varepsilon \Leftrightarrow 0<a=\tilde{a}=h<1$, the second equation of (3.5) uniquely defines

$$
k(a)=\frac{\varepsilon}{\delta} h^{\prime}\left(h^{-1}(a)\right), \quad a \in(0,1) .
$$

Note that $k(0+)=k(1-)=0$ and $k(a)>0, a \in(0,1)$, hold as expected and we can extend $k$ to be continuous in a neighbourhood of $[0,1]$. Furthermore,

$$
k^{\prime}(a) \rightarrow \pm \infty, \quad a \rightarrow\left\{\begin{array}{l}
0+ \\
1-
\end{array},\right.
$$

(provided the limits exist). This can be seen as follows. The equation $\dot{a}=k(a)$ gives formally

$$
\xi+\frac{\delta}{\varepsilon}=\int_{-\delta / \varepsilon}^{\xi} d y=\int_{0}^{a} \frac{d \alpha}{k(\alpha)}
$$

for small $\xi+\delta / \varepsilon>0 \Leftrightarrow$ small $a>0$. If $k^{\prime}(a) \leq C<\infty$ for small $a>0$, then $k(a)=k(0)+k^{\prime}(\theta) a \leq C a$ for small $a>0$, and the last integral in (3.8) would be divergent, which contradicts the fact that the left-hand side is finite.

\subsection{Classification of Waves.}

Consider the system (3.3) with $k(a)$ defined by (3.6) and a shock wave (at $x=0$ ) between $\left(u^{-}, 0\right)$ and $\left(u^{+}, 1\right)$. Depending on $f$ and $g$ and the initial data there are different types of shock according to Cases 1, 2 and 3 after the proof of Proposition 2.1. In the next section we shall see that this is also a natural division with respect to the corresponding viscous profiles. Let us compare with the notion of undercompressive waves, etc. of (3.3). The first eigenvalue of (3.3) satisfies $\lambda_{1}\left(u^{-}, 0\right)=g^{\prime}\left(u^{-}\right)$, $\lambda_{1}\left(u^{+}, 1\right)=f^{\prime}\left(u^{+}\right)$and the second $\lambda_{2}\left(u^{-}, 0+\right)=k^{\prime}(0+)>0, \lambda_{2}\left(u^{+}, 1-\right)=k^{\prime}(1-)<0$. We say that the wave is

undercompressive if $\quad g^{\prime}\left(u^{-}\right)<0, f^{\prime}\left(u^{+}\right)>0$, marginal undercompressive if $g^{\prime}\left(u^{-}\right)=0, f^{\prime}\left(u^{+}\right)>0$ or $g^{\prime}\left(u^{-}\right)<0, f^{\prime}\left(u^{+}\right)=0$, overcompressive if $\quad g^{\prime}\left(u^{-}\right)>0, f^{\prime}\left(u^{+}\right)<0$, marginal overcompressive if $g^{\prime}\left(u^{-}\right)=0, f^{\prime}\left(u^{+}\right)<0$ or $g^{\prime}\left(u^{-}\right)>0, f^{\prime}\left(u^{+}\right)=0$, a regular Lax wave if $\quad g^{\prime}\left(u^{-}\right)<0, f^{\prime}\left(u^{+}\right)<0$ or $g^{\prime}\left(u^{-}\right)>0, f^{\prime}\left(u^{+}\right)>0$, degenerate if $\quad g^{\prime}\left(u^{-}\right)=f^{\prime}\left(u^{+}\right)=0$.

The three main types of shock as well as types of viscous profile are the following, referring to the three cases after the proof of Proposition 2.1.

Case 1 . Here $u^{+}=u^{-}=\bar{u}$, so there is only a discontinuity in $a$. Generally, the wave is undercompressive, but it may also be marginal undercompressive or degenerate. $v(\xi) \equiv \bar{u}$ is the unique viscous profile of (3.2), see Sect. 4 .

Case 2. Generally, the wave is a regular Lax shock, but it may also be marginal under or overcompressive or degenerate. The viscous profile of (3.2) is unique and satisfies $v(\xi) \equiv u^{-}$for $\xi<-\delta / \varepsilon$. If the ratio $\delta / \varepsilon$ is small enough, the profile is increasing for $\xi>-\delta / \varepsilon$. For larger values of $\delta / \varepsilon$ it may be non-monotone in $|\xi|<\delta / \varepsilon$, see 
Sect. 4.

Case 3. Generally, the wave is overcompressive, but it may also be marginal overcompressive or degenerate. There are infinitely many viscous profiles of (3.2), of which some can be non-monotone, see Sect. 4 . The smallness assumption on the ratio $\delta / \varepsilon$ is needed in some cases.

\section{The Equivalence between Condition $\Gamma$ and the Viscous Profile Condition}

A piecewise smooth solution $u$ satisfies $\left(u^{+}, u^{-}\right) \in P\left(u_{+}\right) \times N\left(u_{-}\right)$for all but a finite number of $t^{\prime}$ 's, see [2]. Those events when $\left(u^{+}, u^{-}\right) \notin P \times N$ occur only when moving plateaus of $\hat{f}$ and $\breve{g}$ meet, i.e., when they have the same function value. The movements of the plateaus depend on the initial data. Condition $\Gamma$ resolves this, but the viscous profile condition only considers stationary shocks. The situation is completely analogous to the relation between Oleinik's entropy condition $\left(S\left(u, u_{-}\right) \geq\right.$the shock speed for all $u$ between $u^{-}$and $u^{+}$) and the viscous profile condition for the scalar equation $u_{t}+f(u)_{x}=0$. Condition $\Gamma$ is a generalization of Oleinik's entropy condition, see Sect. 2. Generally, if there exists a viscous profile between two states, then the corresponding discontinuity of the hyperbolic equation fulfils Oleinik's entropy condition in the case $f=g$ and, as we shall prove below, Condition $\Gamma$ in the case of $f \neq g$. However, there are situations when Oleinik's entropy condition/Condition $I$ admits a discontinuity of the corresponding hyperbolic equation, which does not admit a viscous profile.

Example 4.1. The problem

$$
\begin{aligned}
& u_{t}+\left(u^{2}-u^{4}\right)_{x}=\varepsilon u_{x x}, \\
& u(x, 0)=2 H(x)-1
\end{aligned}
$$

has, with $\varepsilon=0$, the stationary entropy solution $u(x, t)=2 H(x)-1, t \geq 0$, but there is no corresponding viscous profile. This is because $\dot{v}=f(v) \geq 0$ for $|v|<1$, with equality for $v=0$. Thus $v(\xi) \equiv 0$ is a solution, and by the uniqueness theorem for ordinary differential equations there does not exist any profile from -1 to 1 , since the graph would then cross the line $v=0$. Hence the solution $u(x, t)=2 H(x)-1$ is unstable when including diffusion. Computer simulations indicate that the solution of (4.1) with $\varepsilon>0$ tends to zero pointwise as $t \rightarrow \infty$. Such situations are avoided by Matsumura and Nishihara [10] and Jones, Gardner and Kapitula [9], by assuming that $S\left(u, u_{-}\right)$is strictly larger than the shock speed for all $u$ strictly between $u^{-}$and $u^{+}$.

Analogous situations occur in the case of a discontinuous flux function and Condition $\Gamma$, see Examples 4.2 and 4.3

Consider the Riemann problem with discontinuous flux function (2.6). The set

$$
\Pi=\left\{(\alpha, \beta) \in P\left(u_{r}\right) \times N\left(u_{l}\right): f(\alpha)=g(\beta)\right\}
$$

contains precisely those pairs for which there exists a piecewise smooth solution of (2.6) with $\left(u^{+}(t), u^{-}(t)\right) \equiv(\alpha, \beta)$. Theorem 2.1 implies that Condition $\Gamma$ picks out the pair $\left(u^{+}, u^{-}\right)=c\left(u_{r}, u_{l}\right)$ of $\Pi$. We shall now show that the viscous profile condition uniquely picks out the same pair. Note that $I\left(u_{r}, u_{l}\right) \neq \emptyset$ implies $\Pi \neq \emptyset$. 
Theorem 4.1. Given $u_{l}$ and $u_{r}$ with $I\left(u_{r}, u_{l}\right) \neq \emptyset$. Let $\left(u^{+}, u^{-}\right)=c\left(u_{r}, u_{l}\right)$ and assume that there exists a viscous profile between $u^{-}$and $u^{+}$. Then Condition $\Gamma$ is equivalent to the viscous profile condition in the following sense: If the ratio $\delta / \varepsilon$ is small enough (see the lemmas below), $\left(u^{+}, u^{-}\right)$is the only pair of $\Pi$ that admits a viscous profile of (3.2).

The theorem will be proved by considering different cases of $f$ and $g$ in the lemmas below. For given $u_{r}, u_{l}, f$ and $g$, the stationary viscous profile obtained below will thus, as $\delta, \varepsilon \searrow 0$ with $\delta / \varepsilon$ bounded, describe the discontinuity along the $t$ axis of the solution of (2.6) satisfying Condition $\Gamma$. From now on it will be convenient to let $\left(u_{p}, u_{m}\right)=c\left(u_{r}, u_{l}\right)$ denote the specific pair in $\Pi$ chosen by Condition $\Gamma$. By symmetry we only consider discontinuities with $u_{m} \leq u_{p}$, and when we write Case 2 in the sequel we mean Case 2a. In Case $1 u_{p}=u_{m}$ holds.

It is convenient to write the non-autonomous Eq. (3.2) as an autonomous system and to introduce the function $V$

$$
\left\{\begin{array}{l}
\dot{v}=h(\varepsilon \xi / \delta)(f(v)-g(v))+g(v)-g\left(u^{-}\right) \equiv V(v, \xi) \\
\dot{\xi}=1
\end{array}\right.
$$

Then the viscous profile is obtained directly in the $\xi-v$ plane.

\subsection{Case 1.}

The general behaviour is captured by the following lemma. Note that in this case $u_{p}=u_{m}=\bar{u}$.

Lemma 4.1. Assume that there is a unique intersection of the graphs of $f$ and $g$ at $\bar{u}$ with

$$
\begin{aligned}
& g(u) \gtrless g(\bar{u})=\gamma \\
& f(u) \lessgtr f(\bar{u})=\gamma
\end{aligned} \quad u \lessgtr \bar{u},
$$

cf. Fig. 5. Independently of $u_{r}$ and $u_{l}$ this is a case- 1 intersection of $\hat{f}$ and $\check{g}$ with $\bar{u} \in \operatorname{int}(N \cap P)$. Then Condition $\Gamma$ is equivalent to the viscous profile condition and there is a unique viscous profile $v(\xi) \equiv \bar{u}$.

Proof. Firstly, let $u^{+}=u^{-}=\bar{u}$ according to Condition $\Gamma$. Then $V(\bar{u}, \xi)=0, \forall \xi$, and

$$
V(v, \xi)=\left\{\begin{array}{l}
g(v)-\gamma \lessgtr 0, \quad \xi<-\delta / \varepsilon \\
f(v)-\gamma \gtrless 0, \quad \xi>\delta / \varepsilon
\end{array}, \quad v \gtrless \bar{u},\right.
$$

which implies the phase portrait (of (4.2)) in Fig. 7. Hence $v(\xi) \equiv \bar{u}$ is the unique solution of (4.2) satisfying $v(\xi) \rightarrow u^{ \pm}=\bar{u}, \xi \rightarrow \pm \infty$.

Secondly, let $\left(u^{+}, u^{-}\right) \in \Pi$ with $u^{-}<\bar{u}$. Then $f\left(u^{+}\right)=g\left(u^{-}\right)$implies $u^{-}<\bar{u}<$ $u^{+}$. Further $V(\bar{u}, \xi)=g(\bar{u})-g\left(u^{-}\right)<0, \forall \xi$, so no orbit can reach $u^{+}$starting from $u^{-}$at $\xi=-\infty$.

The case $u^{-}>\bar{u}$ is analogous.

If $u_{r}, u_{l}, f$ and $g$ are such that (4.3) only holds in a neighbourhood of $\bar{u}$, i.e., $\bar{u} \in \operatorname{int}(N \cap P)$, the lemma still holds and the modifications of the proof are obvious. If $\bar{u} \in \partial(N \cap P)$, e.g. $f(u)=-u^{3}$ and $g(u)=u^{2}$, the result is the same and we omit the details. 


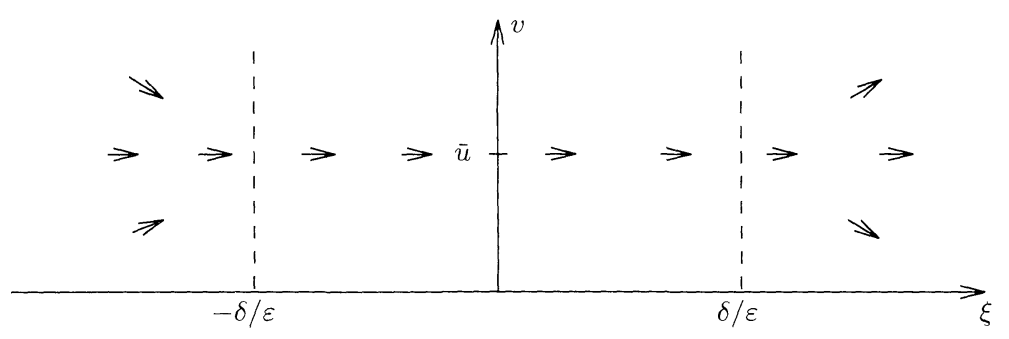

Fig. 7. (Lemma 4.1) Phase plane diagram in the case $u^{+}=u^{--}=\bar{u}$.

\subsection{Case 2.}

We shall see that there exists a unique viscous profile of (4.2), which satisfies $v(\xi) \equiv$ $u_{m}$ for $\xi<-\delta / \varepsilon$.

Lemma 4.2. Consider a case- 2 intersection at $\bar{u}$ with $u_{l}$ such that $\breve{g}\left(u ; u_{l}\right)=g(u)$ is decreasing for $u$ in a neighbourhood of $\bar{u} ; \hat{f}$ has a plateau from $u_{0}$ to $u_{1} ; u_{0}<\bar{u}<$ $u_{1} \leq u_{r} ; f(u)>f\left(u_{0}\right)=f\left(u_{1}\right)=g(\bar{u}) \equiv \gamma, u \in\left(u_{0}, u_{1}\right) ; g(u)<\gamma, u \in\left(\bar{u}, u_{1}\right) ;$ $f(u) \geq \gamma$ for $u>u_{1}$; cf. Fig. 8. Then Condition $\Gamma$ is equivalent to the viscous profile condition. The unique viscous profile is non-decreasing.

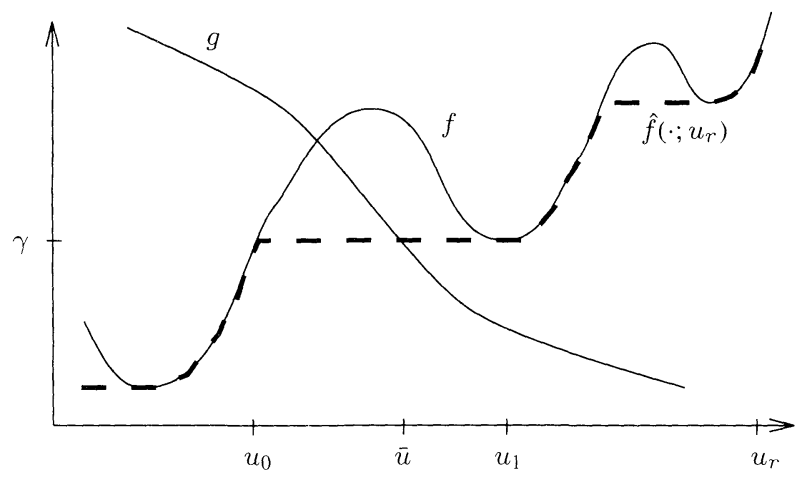

Fig. 8. The flux functions in Lemma 4.2.

Proof. Assume first that $g(u)$ is decreasing for all $u$. Let $\left(u^{+}, u^{-}\right)=\left(u_{1}, \bar{u}\right)=\left(u_{p}, u_{m}\right)$ according to Condition $\Gamma$. We obtain

$$
\begin{aligned}
& V(\bar{u}, \xi)=h(\varepsilon \xi / \delta)(f(\bar{u})-\gamma)\left\{\begin{array}{ll}
=0, & \xi \leq-\delta / \varepsilon \\
>0, & \xi>-\delta / \varepsilon
\end{array},\right. \\
& V\left(u_{1}, \xi\right)=(1-h(\varepsilon \xi / \delta))\left(g\left(u_{1}\right)-\gamma\right)\left\{\begin{array}{ll}
<0, & \xi<\delta / \varepsilon \\
=0, & \xi \geq \delta / \varepsilon
\end{array},\right. \\
& V(v, \xi)=g(v)-\gamma \gtrless 0, \quad \xi \leq-\delta / \varepsilon, \quad v \lessgtr \bar{u}, \\
& V(v, \xi)=f(v)-\gamma>0, \quad \xi \geq \delta / \varepsilon, \quad v \in\left(\bar{u}, u_{1}\right),
\end{aligned}
$$


which gives the phase portrait in Fig. 9. Hence there exists a unique viscous profile

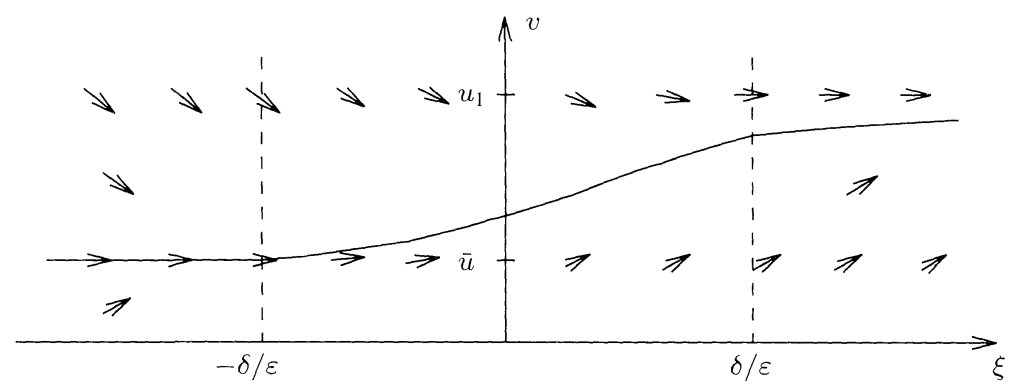

Fig. 9. (Lemma 4.2) Phase plane diagram and the viscous profile in the case when $\left(u^{+}, u^{-}\right)=\left(u_{1}, \bar{u}\right)$.

with $v(\xi)=\bar{u}$ for $\xi \leq-\delta / \varepsilon$ and $v(\xi) \nearrow u_{1}$ as $\xi \rightarrow \infty$.

If $u^{-}<\bar{u}$, then $\left(u^{+}, u^{-}\right) \in \Pi$ implies $u^{+}>u_{1}$. Then

$$
V\left(u_{1}, \xi\right)=(1-h(\varepsilon \xi / \delta))\left(g\left(u_{1}\right)-\gamma\right)+\gamma-g\left(u^{-}\right)<0, \quad \forall \xi,
$$

so no curve $v(\xi)$ can cross the constant line $u_{1}$ from below. Hence there does not exist any viscous profile from $u^{-}$to $u^{+}$.

If $u^{-}>\bar{u}$, then $\left(u^{+}, u^{-}\right) \in \Pi$ implies $u^{+}<u_{0}$. Then there is a $\hat{u} \in\left(u^{+}, u^{-}\right)$such that $f(\hat{u})>g\left(u^{-}\right), g(\hat{u})>g\left(u^{-}\right)$, which implies $V(\hat{u}, \xi)>0, \forall \xi$. Thus there exists no orbit crossing the constant line $\hat{u}$ from above.

If $g(u)$ is decreasing only in a neighbourhood of $\left[\bar{u}, u_{1}\right]$, the same conclusion as above can be made.

The unique viscous profile $v(\xi)$, see Fig. 9, is non-decreasing, because

$$
\frac{\partial V}{\partial \xi}=\frac{\varepsilon}{\delta} h^{\prime}\left(\frac{\varepsilon \xi}{\delta}\right)(f(v)-g(v))\left\{\begin{array}{ll}
=0, & |\xi| \geq \delta / \varepsilon \\
>0, & |\xi|<\delta / \varepsilon
\end{array}, \quad v \in\left(\bar{u}, u_{1}\right)\right.
$$

implies that once the curve $v(\xi)$ has passed a point $(\hat{\xi}, \hat{v})$ with $\dot{v}(\hat{\xi}) \geq 0$, it holds that $V(\hat{v}, \xi)>0, \forall \xi>\hat{\xi}$, so the curve can not cross the horizontal line with value $\hat{v}$ from above.

Note that the wave of Lemma 4.2 is marginal undercompressive if $g^{\prime}\left(u_{m}\right)=$ $g^{\prime}(\bar{u})<0$ and degenerate if $g^{\prime}\left(u_{m}\right)=0$.

Depending on $f, g, u_{r}$ and $u_{l}$ there are several qualitatively different possibilities to obtain a case-2 intersection. However, (4.4) always holds for $v$ close to $u^{-}=\bar{u}$, so that the profile will always be unique. We shall now proceed to other case- 2 intersections without going into every detail, since the proofs only consist in simple phase plane analyses. The phase plane diagrams shown below are obtained by computer simulations, where we have used $\delta / \varepsilon=1$ and $h(x)=(1+\sin (x \pi / 2)) / 2$ for $|x|<1$.

In Lemma 4.2, the values of $f(u)$ for $u>u_{1}$ only influence the phase portrait for $u>u_{1}$. If $u_{r}=u_{1}$ we may assume that $f(u)<\gamma$ for $u>u_{1}$ and the proof above will yield the following lemma.

Lemma 4.3. Given the prerequisites of Lemma 4.2, except that $u_{r}=u_{1}$ and $f$ is decreasing in a neighbourhood of $u_{r}$. Then Condition $\Gamma$ is equivalent to the viscous profile condition. The viscous profile is non-decreasing. 
Since $\hat{f}\left(u ; u_{r}\right)=\gamma$ and $\check{g}\left(u ; u_{l}\right)=g(u)$ for $u$ in a neighbourhood of $\left[\bar{u}, u_{r}\right]$, we have $\left(u^{+}, u^{-}\right)=\left(u_{p}, u_{m}\right)=\left(u_{r}, \bar{u}\right)$. The phase portrait will be qualitatively as in Fig. 10.
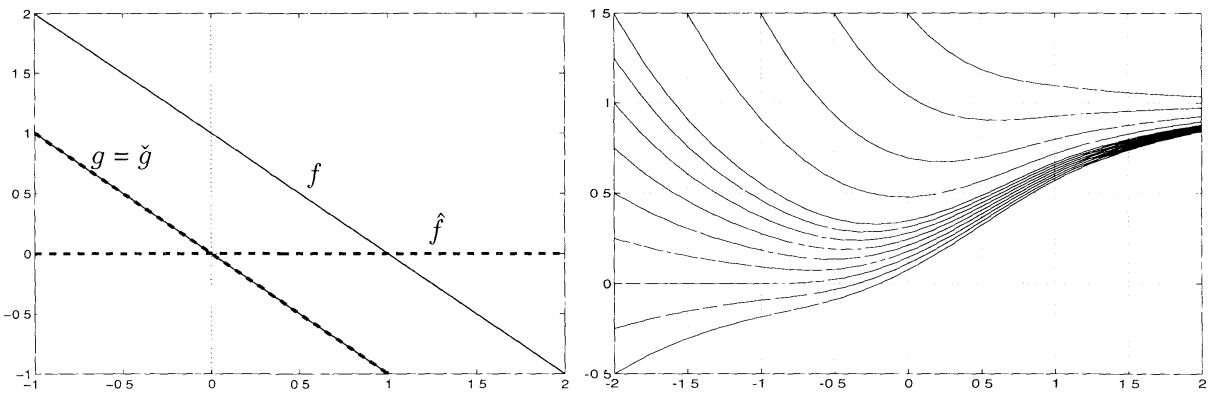

Fig. 10. (Lemma 4.3) Phase portrait (right) when $g(u)=-u, f(u)=1-u, u^{-}=u_{m}=0$ and $u^{+}=u_{p}=1$.

Lemma 4.4. Given the prerequisites in Lemma 4.2 or Lemma 4.3, but relax the assumption $g(u)<\gamma, u \in\left(\bar{u}, u_{1}\right)=\left(u_{m}, u_{p}\right)$. If the ratio $\delta / \varepsilon$ is so small that

$$
\max _{|x| \leq 1, u_{m} \leq u \leq u_{p}}|h(x)(f(u)-g(u))+g(u)-\gamma|<\frac{\varepsilon}{2 \delta}\left(u_{p}-u_{m}\right)
$$

then Condition $\Gamma$ is equivalent to the viscous profile condition.

Remark. (4.5) is only needed if both $g(u)>\gamma$ and $f(u)>\gamma$ for small $u-u_{p}>0$.

Proof. Assume that $g(u)>\gamma$ for some $u \in\left(\bar{u}, u_{1}\right)$, otherwise one of the two previous lemmas applies. Let first $\left(u^{+}, u^{-}\right)=\left(u_{p}, u_{m}\right)$ according to Condition $\Gamma$. If $f(u)<\gamma$ for small $u-u_{p}>0$, the interesting part of the phase plane is qualitatively as in Fig. 11 or 12 . Hence there is a unique profile from $u^{-}=u_{m}$ to $u^{+}=u_{p}$ independently
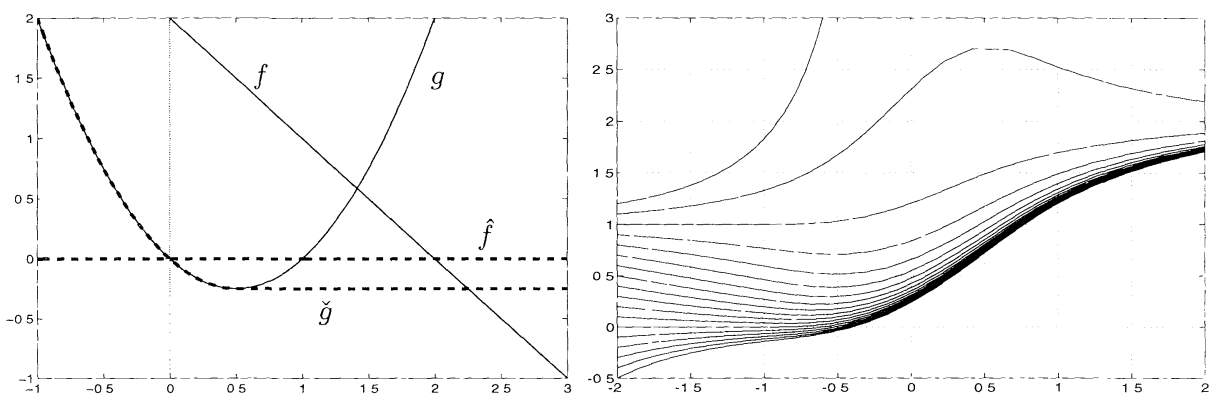

Fig. 11. (Lemma 4.4) Phase plane diagram (right) when $g(u)=u(u-1), f(u)=2-u, u^{-}=u_{m}=0$ and $u^{+}=u_{p}=2$.

of the ratio $\delta / \varepsilon$. If $f(u)>\gamma$ for $u>u_{p}$, then $V(v, \xi)=f(v)-\gamma>0$ for $v>u_{p}$, $\xi>\delta / \varepsilon$, hence the orbits with over-shoot in Fig. 12 tend to infinity. However, invoking (4.5), $\dot{v}$ is so small that the orbit satisfying $v(\xi)=u^{-}$for $\xi<-\delta / \varepsilon$ will satisfy $v(\delta / \varepsilon)<u^{+}$, hence $v(\xi) \nearrow u^{+}$as $\xi \rightarrow \infty$ (as in Fig. 11). 

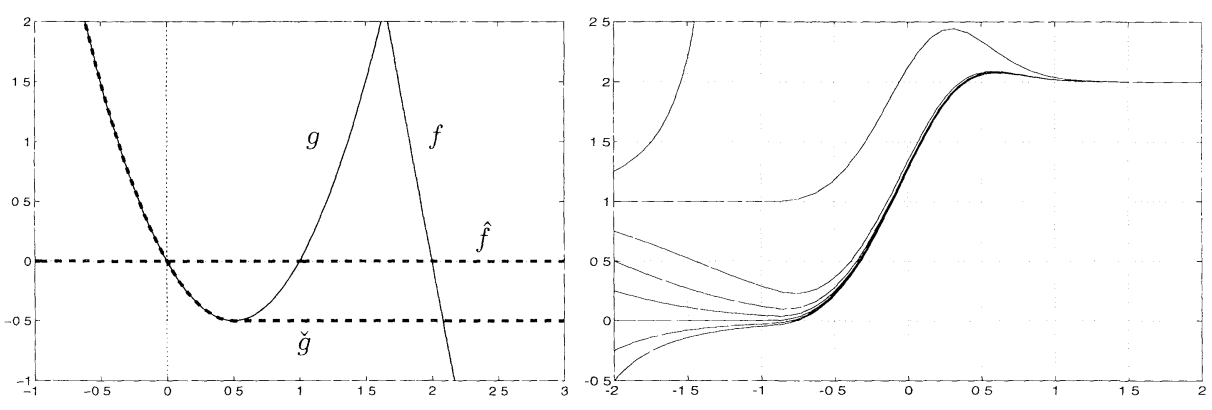

Fig. 12. (Lemma 4.4) Phase plane diagram (right) when $g(u)=2 u(u-1), f(u)=6(2-u), u^{-}=u_{m}=0$ and $u^{+}=u_{p}=2$. Note that the viscous profile is non-monotone. The corresponding shock is a regular Lax wave.

Now consider the other pairs of $\Pi$. The case $u^{-}>u_{m}$ is treated in the proof of Lemma 4.2. Assume that $u^{-}<u_{m}$ and $f(u)>\gamma$ for some $u>u_{p}$ (otherwise this is an empty case). Then $\left(u^{+}, u^{-}\right) \in \Pi$ implies that $u^{+}>u_{p}$. Requiring (4.5), then for the orbit satisfying $v_{1}(\xi) \equiv u^{-}$for $\xi<-\delta / \varepsilon$ it holds that $\dot{v}_{1}(\xi)<\varepsilon / \delta\left(u_{p}-u_{m}\right)<$ $\varepsilon / \delta\left(u^{+}-u^{-}\right)$. Hence $v_{1}(\delta / \varepsilon)<u^{+}$, and it is easy to see that all orbits which are $<u^{+}$at $\xi=\delta / \varepsilon$ tend to $u_{p} \neq u^{+}$as $\xi \rightarrow \infty$.

Before ending this subsection, we give examples of unstable discontinuities. They should be compared to Example 4.1.

Example 4.2. Let the initial data be $u_{0}(x)=2 H(x)$ and the flux functions $g(u)=-u$ and $f(u)=(u-1)^{2}(u-2)^{2}$, see Fig. 13. Here $I I=\{(\alpha, \beta): \alpha \geq 2, \beta \leq 0, f(\alpha)=$
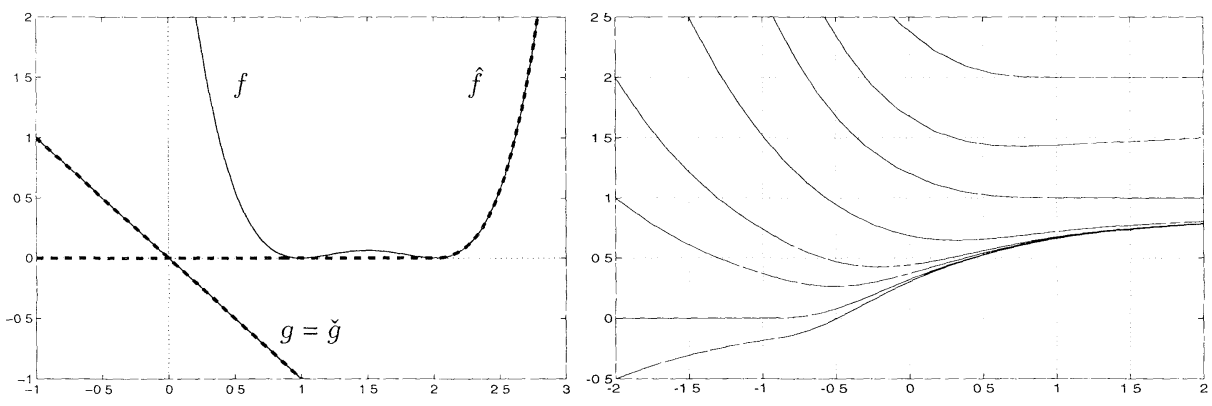

Fig. 13. Phase plane diagram (right) in Example 4.2 .

$g(\beta)\} \cdot u(x, t) \equiv 2 H(x)$ is a solution satisfying Condition $\Gamma$. There exists no viscous profile between 0 and 2 , since $f(1)=0$ implies $V(1, \xi)=h(\varepsilon \xi / \delta)-1 \leq 0$. The exclusions of the existence of viscous profiles for all the other pairs of $\bar{\Pi}$ can be made as in the proof of Lemma 4.2.

Example 4.3. A simpler example than the previous one is obtained by letting $f(u)<\gamma$ for $u>2$, e.g. $f(u)=(u-1)^{2}(2-u)$. Then $\hat{f}(\cdot ; 2) \equiv 2$ and $\Pi=\{(2,0)\}$. Hence Condition $\Gamma$ is not needed since $u(x, t) \equiv 2 H(x)$ is the only possible solution. With 
the same argument as above we conclude that there exists no viscous profile between 0 and 2.

Situations as in the examples above may occur when $\hat{f}$ (or $\check{g}$ ) has a plateau at the level $\gamma$ and there is a $\hat{u} \in\left(u_{m}, u_{p}\right)$ with $f(\hat{u})=\gamma$. The corresponding discontinuities are thus unstable when including diffusion.

\subsection{Case 3.}

In this case one plateau of $\check{g}$ and one of $\hat{f}$ lie on the same flux value level $\gamma=\hat{f}\left(\bar{U} ; u_{r}\right)$. Recall that $\left(u_{p}, u_{m}\right)=c\left(u_{r}, u_{l}\right)$ is the pair chosen by Condition $\Gamma$. In Case 2 precisely one plateau was involved in the intersection. We chose one of $\hat{f}$ in the previous subsection (corresponding to Case $2 \mathrm{a}$ ) and saw that infinitely many orbits converged to $u_{p}$ as $\xi \rightarrow \infty$. The behaviour of the profiles for $\xi>\delta / \varepsilon$ will thus now also hold for $\xi<-\delta / \varepsilon$. Hence there will be infinitely many profiles from $u_{m}$ to $u_{p}$. Because of this symmetry between $+\infty$ and $-\infty$, we shall start by letting $g$ be as simple as possible and consider different $f$ 's, such that a case- 3 intersection is produced. In some cases we need the smallness condition (4.5) on the ratio $\delta / \varepsilon$, which guarantees the existence of monotone profiles from $u_{m}$ to $u_{p}$.

Lemma 4.5. Assume that both $f(u)>\gamma$ and $g(u)>\gamma$ for $u \in\left(u_{m}, u_{p}\right)$. If (4.5) holds, then Condition $\Gamma$ is equivalent to the viscous profile condition.

Remark. (4.5) is only needed if $g(u)>\gamma$ for small $u_{m}-u>0$ and $f(u)>\gamma$ for small $u-u_{p}>0$.

Proof. Let first $\left(u^{+}, u^{-}\right)=\left(u_{p}, u_{m}\right)$ according to Condition $\Gamma$. The qualitative behaviour of the interesting part of the phase plane, when $g(u)<\gamma$ for small $u_{m}-u>0$, is shown in Figs. 14 and 15.
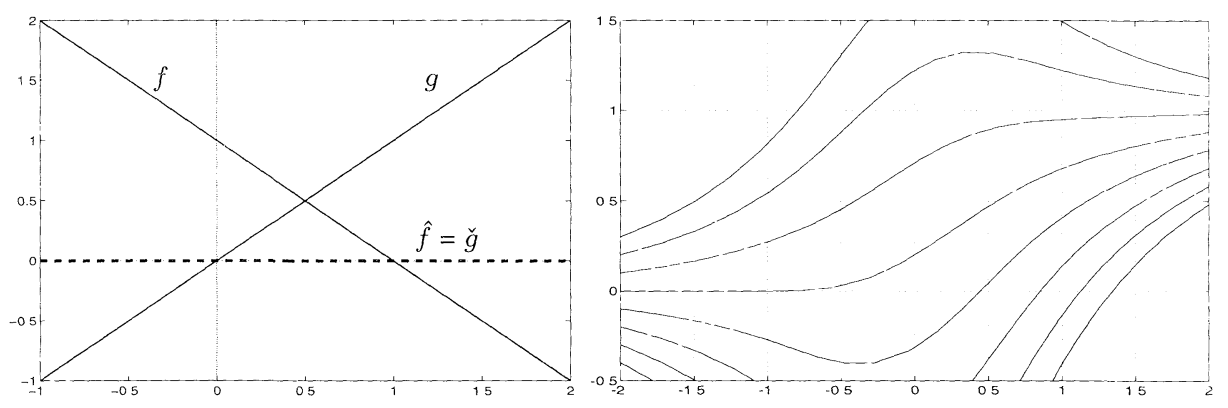

Fig. 14. (Lemma 4.5) Phase plane diagram (right) when $g(u)=u, f(u)=1-u, u^{-}=u_{m}=0$ and $u^{+}=u_{p}=1$. All orbits are viscous profiles. The corresponding shock wave is overcompressive.

If $u^{-}<u_{m}$, then the requirement $\left(u^{+}, u^{-}\right) \in \Pi$ implies $u^{+}>u_{p}$. Then $V\left(u^{i}, \xi\right) \equiv$ 0 , where $u^{i} \in\left(u_{m}, u_{p}\right)$ is an intersection of $f$ and $g$. Hence there exists no profile from $u^{-}$to $u^{+}$. The case $u^{-}>u_{m}$ is analogous.

If the conditions in the remark hold, (4.5) guarantees that the profile satisfying $v(\xi)=u_{m}$ for $\xi<-\delta / \varepsilon$ will increase to $u_{p}$. 

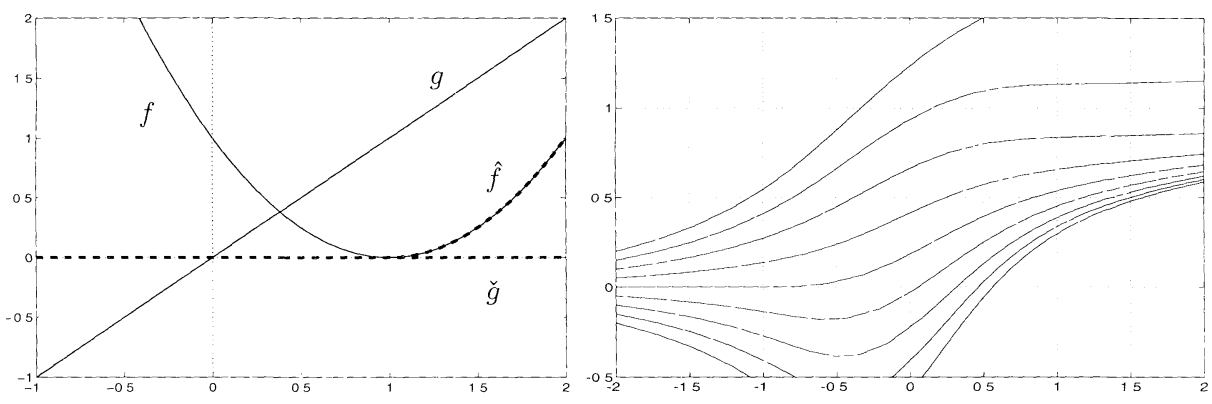

Fig. 15. (Lemma 4.5) Phase plane diagram (right) when $g(u)=u, f(u)=(1-u)^{2}, u^{-}=u_{m}=0$ and $u^{+}=u_{p}=1$. The corresponding shock wave is marginal overcompressive.

Now, we proceed by relaxing the restriction in Lemma 4.5 that the graph of $f$ should lie strictly above the level $\gamma$ on the whole interval $\left(u_{m}, u_{p}\right)$.

Lemma 4.6. Assume that $g(u)>\gamma$ for $u \in\left(u_{m}, u_{p}\right), f\left(u_{0}\right)=0$ for some $u_{0} \in$ $\left(u_{m}, u_{p}\right)$ and $f(u)>\gamma$ for $u \in\left(u_{0}, u_{p}\right)$. If (4.5) holds, then Condition $\Gamma$ is equivalent to the viscous profile condition.

Remark. (4.5) is only needed if $g(u)>\gamma$ for small $u_{m}-u>0$ and $f(u)>\gamma$ for small $u-u_{p}>0$.

Proof. Let first $\left(u^{+}, u^{-}\right)=\left(u_{p}, u_{m}\right)$ according to Condition $\Gamma$. The qualitative behaviour when $f(u)<\gamma$ for small $u-u_{p}>0$ and $g(u)<\gamma$ for small $u_{m}-u>0$ is given by the two phase planes in Figs. 16 and 17. If both hold (see the remark), (4.5) is required. If instead $f(u)>\gamma$ for small $u-u_{p}>0$, then the orbits in $\xi>\delta / \varepsilon$
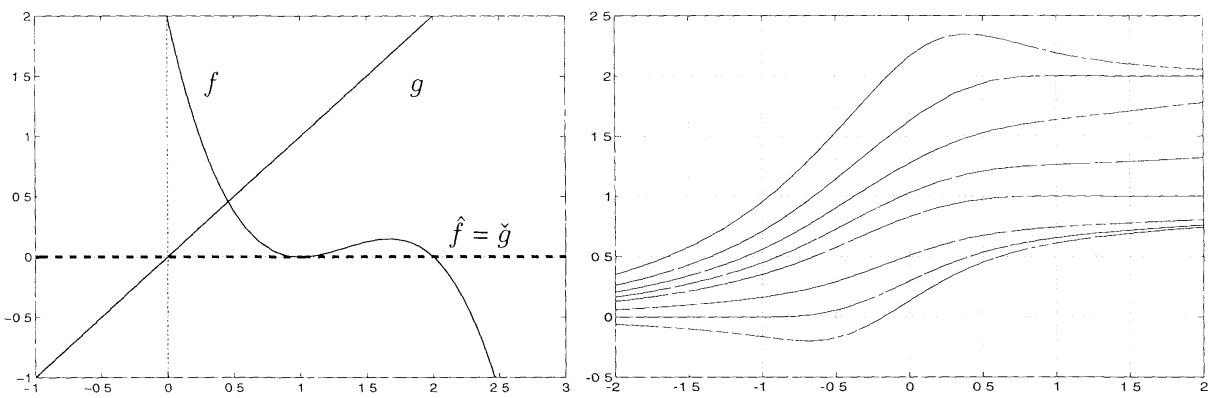

Fig. 16. (Lemma 4.6) Phase plane diagram (right) when $g(u)=u, f(u)=(u-1)^{2}(2-u), u^{-}=u_{m}=0$ and $u^{+}=u_{p}=2$.

just above $v=u_{p}$ would increase. Similarly, if $g(u)>\gamma$ for small $u_{m}-u>0$, this would only affect the phase portrait below $v=u_{m}$ and $\xi<-\delta / \varepsilon$. In any case, it follows that all orbits $v(\xi)$ passing through the points $\xi=\delta / \varepsilon, u_{0}<v(\xi) \leq u_{p}$ are viscous profiles.

Now assume that $u^{-}<u_{m}$. Then the requirement $\left(u^{+}, u^{-}\right) \in \Pi$ implies that $u^{+}>u_{p}$. If the graph of $f$ intersects $g$ at some $u^{\imath} \in\left(u^{-}, u^{+}\right)$, then we have the 

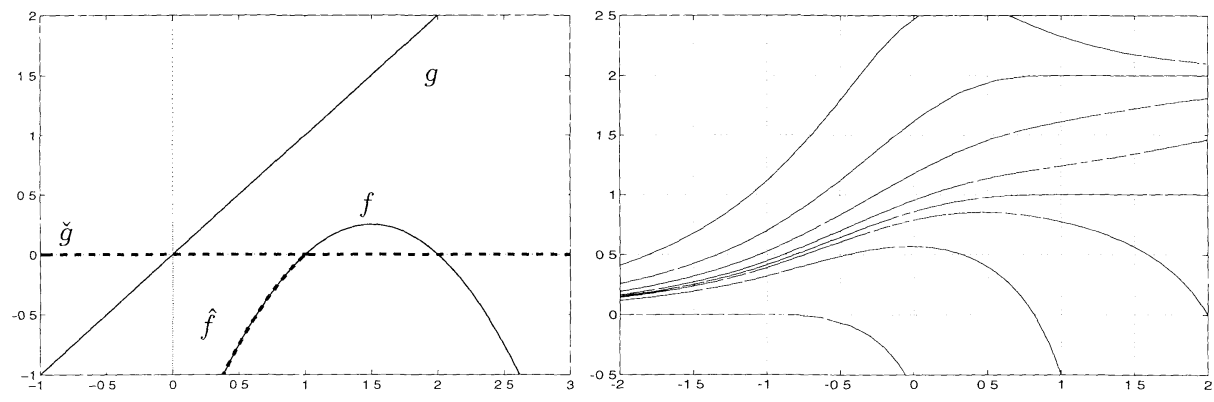

Fig. 17. (Lemma 4.6) Phase plane diagram (right) when $g(u)=u, f(u)=(1-u)(u-2), u^{-}=0$ and $u^{+}=2$.

same case as in the previous proof. Otherwise $f\left(u_{m}\right)<g\left(u_{m}\right)<g\left(u^{-}\right)$holds, which implies $V\left(u_{m}, \xi\right)<0 \forall \xi$, so no orbit can pass the horizontal line $v=u_{m}$ from below.

Finally, assume that $u^{-}>u_{m}$, then $u^{+}<u_{p}<u^{-}$, which implies $V\left(u_{p}, \xi\right)>$ $0 \forall \xi$. Hence no orbit can pass the horizontal line $v=u_{p}$ from above.

We shall now relax the condition on $f$ further and assume that the graph lies below the constant line $\gamma$ in a left neighbourhood of $u_{p}$. In order to have a case- 3 intersection, it follows that $f$ must lie below $\gamma$ also in a right neighbourhood of $u_{p}$.

Lemma 4.7. Assume that $g(u)>\gamma$ for $u \in\left(u_{m}, u_{p}\right)$ and that $f$ has a strict local maximum at $u_{p}$. Then Condition $\Gamma$ is equivalent to the viscous profile condition. There are precisely one monotone and infinitely many non-monotone profiles.

Proof. The assumptions imply that $u_{p}=u_{r}$. Phase plane analysis yields that, for $\left(u^{+}, u^{-}\right)=\left(u_{r}, u_{m}\right)$, there are infinitely many profiles $\searrow u^{-}$as $\xi \rightarrow-\infty$ and $\searrow u^{+}$ as $\xi \rightarrow \infty$, cf. Fig. 18. The profile satisfying $v(\xi)=u_{p}$ for $\xi \geq \delta / \varepsilon$ is monotone.
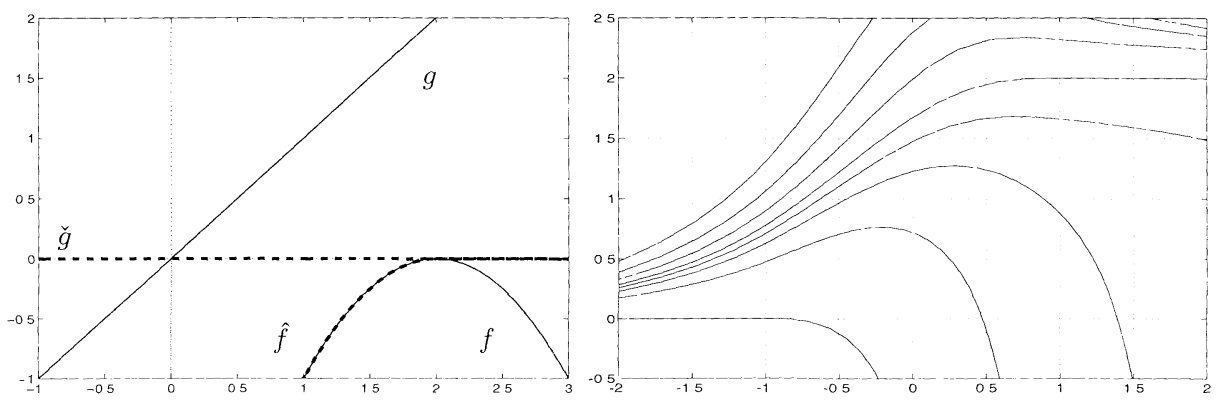

Fig. 18. (Lemma 4.7) Phase plane diagram (right) when $g(u)=u, f(u)=-(u-2)^{2}, u^{-}=u_{m}=0$ and $u^{+}=u_{p}=2$.

Exclusion of other pairs of $\Pi$ can be done exactly as in the proof of Lemma 4.5.

Now we shall relax the condition that $g$ lies strictly above the level $\gamma$ in the interval $\left(u_{m}, u_{p}\right)$. Let $u_{m}<u_{1}<u_{2} \ldots<u_{k}<u_{p}$. Assume that $g\left(u_{j}\right)=0$ for $j=1, \ldots, k$. These points must be local minima, otherwise there is no case- 3 intersection. If $f$ is 
as in the previous lemma, then $V\left(u_{j}, \xi\right) \leq 0$ for all $\xi$. Hence there exists no viscous profile between $u_{m}$ and $u_{p}$, cf. Example 4.2. In fact, $V\left(u_{1}, \cdot\right) \leq 0$ holds for every $f$ satisfying $f\left(u_{1}\right) \leq \gamma$. The only way to obtain a case- 3 intersection which admits a viscous profile from $u_{m}$ to $u_{p}$ is to let $f(u)>\gamma$ at least for $u \in\left(u_{1}, u_{p}\right)$. With this in mind we give the last lemma.

Lemma 4.8. Let $u_{m}<u_{1}<u_{2} \ldots<u_{k}<u_{p}$. Assume that $g(u) \geq \gamma$ for $u \in$ $\left(u_{m}, u_{k}\right)$ with $g\left(u_{j}\right)=0$ for $j=1, \ldots, k$. Assume further that $f(u)>\gamma, u \in\left(u_{0}, u_{p}\right)$ for some $u_{0}<u_{1}$. If (4.5) holds, then Condition $\Gamma$ is equivalent to the viscous profile condition.

Proof. Assume that $u_{0}>u_{m}$, otherwise a symmetric case of Lemma 4.6 applies, cf. Fig. 16. Let $\left(u^{+}, u^{-}\right)=\left(u_{p}, u_{m}\right)$. The qualitative behaviour is given by Fig. 19 . Note that the values of $g(u)$ for $u>u_{k}$ do not affect the interesting orbits. Since the
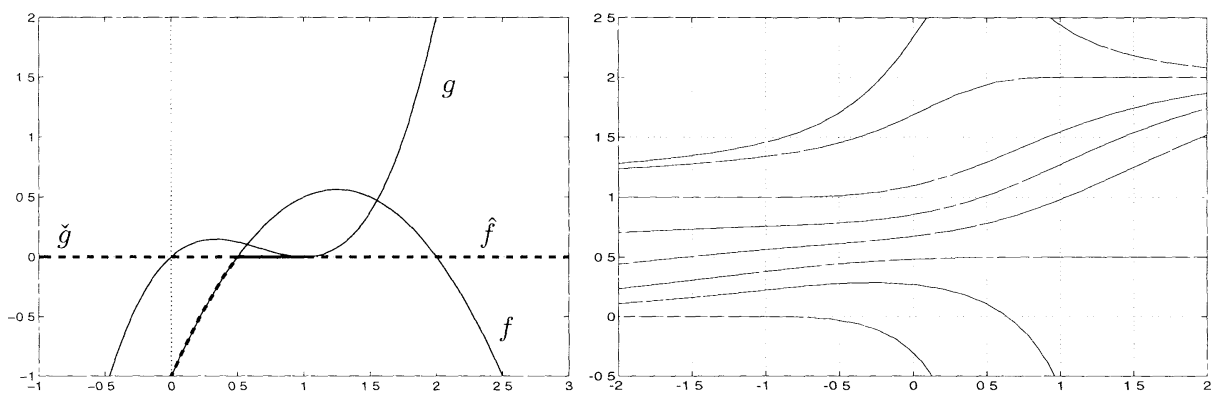

Fig. 19. (Lemma 4.8) Phase plane diagram (right) when $g(u)=u(u-1)^{2}, f(u)=(0.5-u)(u-2)$, $u^{-}=u_{m}=0, u^{+}=u_{p}=2, u_{0}=0.5$ and $u_{1}=1$.

graphs of both $f$ and $g$ lie above the line $v=\gamma$ in the interval $\left(u_{0}, u_{1}\right)$, it follows that $V(u, \xi)>0$ in the rectangle $|\xi|<\delta / \varepsilon, u_{0} \leq u \leq u_{1}$. We claim that each orbit passing through a given point in this rectangle is a viscous profile, which is monotone. Firstly, $V(u, \xi)>0$ in both strips $\xi<-\delta / \varepsilon, u_{m}<u<u_{1}$ (called left strip below) and $\xi>\delta / \varepsilon, u_{0}<u<u_{p}$ (called right strip below). Secondly, (4.5) ensures that the orbit, say $v_{1}(\xi)$, that coincides with the upper horizontal boundary of the left strip, i.e. $v_{1}(\xi)=u_{1}$ for $\xi \leq-\delta / \varepsilon$ (hence passing through the upper left corner of the rectangle), goes into the right strip. Analogously, the orbit with $v_{2}(\xi)=u_{0}$ for $\xi \geq \delta / \varepsilon$ comes from the left strip. Consequently, every orbit passing through the rectangle must lie in both these strips, since it cannot cross neither $v_{1}(\xi)$ nor $v_{2}(\xi)$. Note that if $u_{0}=u_{1}$, the rectangle deteriorates, but there are still infinitely many viscous profiles. Exclusion of other pairs of $\Pi$ can be made as in the previous lemmas.

If $f$ also exhibits the analogue local minima as $g$ in the lemma, it should now be clear that the technique above can be used to establish the desired equivalence.

\subsection{A Remark when $\delta=0, \varepsilon>0$.}

As mentioned in the introduction, $\delta>0$ may provide better models than $\delta=0$ in the applications. Above we have let $\delta>0$, so that the viscous profiles become smooth. 
We have also seen that in many cases there is a boundedness condition on the ratio $\delta / \varepsilon$. The analysis of the equivalence between the uniqueness conditions could have been made in the special case $\delta=0$ and $\varepsilon>0$. Then the region between $|\xi| \leq \delta / \varepsilon$ in the figures above disappears and the viscous profiles become continuous with a jump in their derivative at $x=0$. This can be seen as follows. Let $u_{l}$ and $u_{r}$ denote the states on either side of a discontinuity at $x=0$. Defining $u$ as a weak solution of the equation

$$
u_{t}+(H(x) f(u)+(1-H(x)) g(u))_{x}=\varepsilon u_{x x},
$$

it can be shown (by a standard procedure taking a test function with support near the $t$-axis) that

$$
\begin{array}{ll}
u_{t}+g(u)_{x}=\varepsilon u_{x x}, & x<0, \\
u_{t}+f(u)_{x}=\varepsilon u_{x x}, & x>0, \\
\varepsilon\left(u^{+}-u^{-}\right)=0, & \\
f\left(u^{+}\right)-\varepsilon u_{x}^{+}=g\left(u^{-}\right)-\varepsilon u_{x}^{-} . &
\end{array}
$$

Suppose $u(x, l)=v(x / \varepsilon)$ is a viscous profile with $v(\xi) \rightarrow u_{l}\left(u_{r}\right)$ as $\xi \rightarrow-\infty(\infty)$. Then it follows that

$$
\begin{array}{ll}
\dot{v}=g(v)-g\left(u_{l}\right), & x<0, \\
\dot{v}=f(v)-f\left(u_{r}\right), & x>0, \\
v^{+}=v^{-}, & \\
f\left(v^{+}\right)-\dot{v}^{+}=g\left(v^{-}\right)-\dot{v}^{-}, &
\end{array}
$$

where the last equation can be written $f\left(u_{r}\right)=g\left(u_{l}\right)$. Hence (4.6) is equivalent to (3.2) with $\delta \searrow 0, u^{+} \equiv u_{r}$ and $u^{-} \equiv u_{l}$.

\section{References}

1. Chancelier, J.-Ph., Cohen de Lara, M., and Pacard, F.: Analysis of a conservation pde with discontinuous flux: A model of settler. SIAM J. Appl. Math. 54(4), 954-995 (1994)

2. Diehl, S.: On scalar conservation laws with point source and discontinuous flux function. To appear in SIAM J. Math. Anal., 1995

3. Diehl, S.: A conservation law with point source and discontinuous flux function modelling continuous sedimentation. To appear in SIAM J. Appl. Math., 1996

4. Diehl, S., and Wallin, N.-O.: Scalar conservation laws with discontinuous flux function: II. On the stability of the viscous profiles. To appear in Commun. Math. Phys.

5. Gimse, T., and Risebro, N. H.: Riemann problems with a discontinuous flux function. In: Engquist, B., and Gustavsson, B., editors, Third International Conference on Hyperbolic Problems, Theory, Numerical Methods and Applications, volume I, 1990, pp. 488--502

6. Gimse, T., and Risebro, N. H.: Solution of the Cauchy problem for a conservation law with a discontinuous flux function. SIAM J. Math. Anal. 23(3), 635-648 (1992)

7. Godunov, S. K.: A finite difference method for the numerical computations of discontinuous solutions of the equations of fluid dynamics. Mat. Sb. 47, 271-306 (1959)

8. Holden, L., and Høegh-Krohn, R.: A class of $N$ nonlinear hyperbolic conservation laws. J. Differential Equations 84, 73-99 (1990)

9. Jones, C. K. R. , Gardner, R., and Kapitula, T.: Stability of travelling waves for non-convex scalar viscous conservation laws. Comm. Pure Appl. Math. 46, 505-526 (1993) 
10. Matsumura, A., and Nishihara, K: Asymptotic stability of travelling waves for scalar conservation laws with non-convex nonlinearity. Commun. Math. Phys. 165, 83-96 (1994)

11. Mochon, S: An analysis of the traffic on highways with changing surface conditions. Math. Model. 9(1), 1-11 (1987)

12. Oleinık, O. A. : Uniqueness and stability of the generalized solution of the Cauchy problem for a quasi-linear equation. Uspekhi Mat. Nauk 14, 165-170 (1959) Amer. Math. Soc. Transl. Ser. 2 33, 285-290 (1964)

13. Ross, D. S.: Two new moving boundary problems for scalar conservation laws. Comm. Pure Appl. Math. 41, 725-737 (1988)

\section{Communicated by H. Araki}

This article was processed by the author using the $\mathrm{IAT}_{\mathrm{E}} \mathrm{X}$ style file pljourl from Springer-Verlag. 